Archived version from NCDOCKS Institutional Repository - http://libres.uncg.edu/ir/asu/

Spaulding, T.J., Furukawa, M., Raghu, T.S., and Vinze, A. (2013) Event sequence modeling of IT adoption in healthcare. Decision Support Systems, 55(2): 428-437 (May 2013). Published by Elsevier (ISSN: 1873-5797). The version of record is available from:

http://dx.doi.org/10.1016/j.dss.2012.10.002

\title{
Event sequence modeling of IT adoption in healthcare
}

Trent J. Spaulding, Michael F. Furukawa, T.S. Raghu, and Ajay Vinze

\begin{abstract}
Information systems research is replete with examples of the importance of business processes defining IT adoption. Business processes are influenced by both organizational and operational concerns. We evaluate the comparative importance of operational and organizational influences for complementary IT systems. In the context of acute-care hospitals the analysis shows that an organizational approach to automating a process is related to different financial outcomes than an operational approach. Six complementary systems supporting a three-stage medication management process are studied: prescribing, dispensing, and administration. The analysis uses firm-level, panel data extracted from the HIMSS Analytics database spanning ten years of IT adoption for 140 hospitals. We have augmented the HIMSS dataset with matching demographic and financial details from the American Hospital Association and the Centers for Medicare and Medicaid Services. Using event sequence analysis we explore whether organizations are more likely to adopt organization boundary spanning systems and if the sequence of adoption follows the temporal ordering of the business process steps. The research also investigates if there is a relationship between the paths to IT adoption and financial performance. Comparison of the two measures suggests that the organizational model of adoption is observed more often in the data. Following the organizational model of adoption is associated with approximately $\$ 155$ dollar increase in net income per patient day; whereas the operational model of adoption is associated with approximately $\$ 225$ dollars decrease in net income per patient day. However, this effect diminishes with the adoption of each additional system thus demonstrating that the adoption path effects may only be relevant in the shortterm.
\end{abstract}




\section{INTRODUCTION}

Health care costs and efficiency concerns continue to dominate healthcare policy discussions despite numerous regulations and reforms. In response to a number of recent initiatives and regulations, hospitals have been investing in Health Information Technologies (HIT) to address cost and efficiency concerns. With a variety of efforts underway, the impact of these investments remains unclear. More recently, a number of studies have begun to show that HIT can actually increase costs and reduce efficiencies in hospitals [17], [18] and [19]. Interestingly, there is wide variation in adoption across technologies, hospital characteristics, and geographic locations [20]. These variations in adoptions suggest that hospitals may be using distinct strategies in HIT investments to address costs and efficiencies. We explore this possibility using a theoretical rationale from the business process management literature to understand HIT adoption patterns in hospitals and the resulting impact on performance. The analysis adapts an event sequencing approach to model and identify distinct adoption strategies among hospitals.

The objective of this research is to explore differences in technology adoption patterns in a healthcare setting and to explore the relationship that adoption patterns may have with financial performance. In addressing this objective this paper makes several contributions. The first is a unique application of the underlying business process to dictate relationships between applications. The second contribution is the development of organizational and operational models of process automation. The third contribution is, the novel application of sequence analysis and the Levenshtein distance to information systems research and the order of adoption of IS. Finally, this research has implications in regards to healthcare IT adoption and the organizational fit of long-term IT strategy.

Business processes create the organizational and operational contexts for adoption of IT innovations [36]. As a result, both academic and practitioner literatures emphasize the need for aligning IT strategy with business strategy [3]. When IT and business strategies are aligned, complementarities and synergies from adopting IT systems can be enhanced. While there are strong arguments for alignment, achieving alignment continues to be a challenge in organizations. The primary reason for this is that business processes typically cut across divisional and functional boundaries where power and politics may create challenges and conflict among stakeholders [29]. The contingent nature of these organizational challenges can motivate organizations to follow different paths of IT systems adoption.

Though adoption of interrelated IT applications has been studied in the literature, the use of a business process orientation to understand adoption of technology in this context is not sufficiently documented. To the best of our knowledge, no studies exist in IS literature that has specifically considered IT adoption paths and the impact of those paths on financial performance. Previous research in 
other industries has empirically documented that there are strong correlations in technology adoption decisions in organizations. In the transportation industry, Golob and Regan [23] studied adoption of seven different IT systems and discovered tendencies for bundled IT adoptions in firms. However, their work did not investigate paths of adoption nor did it investigate related performance impacts. Smith and Weil [38] investigated the possibility of adopting manufacturing technologies in sequence in the context of retail industry. The empirical study examined adoption of barcoding, order processing, distribution and assembling technologies. Although Smith and Weil [38] found complementary effects of multiple system adoptions, the sequential nature of adoption could not be completely examined due to the limitations of the data. Battisti et al. [8] investigated joint adoption of IT equipment and innovative work practices in Italian metalworking plants. These studies found that complementarity increases probability of adoption of these technologies. None of these prior works however directly address business process boundary issues in the sequential adoption of complementary technologies.

With an intention to develop an understanding of the effect of business processes on the adoption of complementary systems, we propose two models of adoption as outcomes of operational and organizational influences.

Organizational influences refer to factors such as organizational structure, politics and culture. Operational influences refer to variance and cost control through automation and integration. We describe a clinical care process in the hospital setting, and associated information systems. The process is used to propose reference sequences. Healthcare and hospitals provide a rich basis for the study of adoption of complementary technologies since the underlying business and decisional processes are tightly integrated. The process of ordering a drug, verifying the order, dispensing the order and administering the order are well defined and consistent across hospitals for patient safety and regulatory reasons. This homogeneity in the business process allows us to observe whether organizational or operational themes drive adoption decisions across different facilities.

Extant literature on adoption focuses on the precursors of adoption such as user characteristics, user and group resistance (e.g. [15], [21], [27] and [40]), or power and politics [29]. We specifically address the outcomes of adoption. Literature on the value of IT both in healthcare and more generally focuses on the value of an individual system (e.g. [16]), aggregate measures of IT (e.g. [32]) or sets of systems which do not account for interactions (e.g. [14]). This study examines how different paths of adoption can lead to different financial returns for the organization and suggests that there are interactions between the systems. Angst et al. [4] recently published a paper closely related to this study. They posit that integration is a key factor in the value creation of systems at the Enterprise level. Using a cluster analysis they discover several key patterns. They found that order of adoption did have an effect on cost and quality within the hospital. Their work observed a wide range of systems, both clinical and administrative, 
within the hospital. This research, in contrast, posits that the business process is the key to discovering points of integration. Therefore this study focuses on the systems within one process and uses the process to define key operational and organizational factors.

The next section of this paper uses relevant literature to construct two models of adoption. Section 3 describes the context of the study and operationalizes the models of adoption as hypothesized sequences of adoption. Section 4 presents the data and a detailed description of how the sequence analysis works. Section 5 includes the actual regressions and results. Section 6 discusses implications for IT decision makers and for the healthcare industry.

\section{PATTERNS FOR ADOPTION OF COMPLEMENTARY SYSTEMS}

Two models of adoption are developed using the business process context. An operational perspective on IT adoption in business processes implies a focus on task automation primarily intended to address time and cost savings [24], [25] and [35]. On the other hand, both practitioners and academics also advocate the realignment of roles and responsibilities and organizational structures as other enablers of process improvement [12], [24] and [37]. These two orientations are not mutually exclusive.

\subsection{Patterns of adoption from an organizational perspective}

A business process has organizational characteristics in that it often crosses organizational boundaries. Automation of a process often alters the balance and distribution of power within the organization. As a result, organizational politics and power become significant factors in the adoption of IT innovations into organizations [29] and [30]. Because of these organizational issues, managers find it more difficult to implement systems or interfaces between systems that cut across organizational boundaries than to implement systems that are within the organizational boundary.

Discussion of the effects of the organizational environment of business processes on IT innovation adoption requires that we take a political view similar to Markus [29]. Markus argues that the political view in relation to information systems is most relevant when (1) stakeholders disagree on the nature of the problem, (2) stakeholders disagree about the ability of the system to solve the problem and (3) power is valued and scarce. Disagreements on the nature of the problem, disagreement on the ability of the system to solve the problem and power struggles are more likely to occur when managers and decision makers belong to different organizational units. Thus it is more likely that power struggles and disagreement will exist when decisions are made between organizational units than when they are made within an organizational unit. 
The organization model takes the path of least organizational resistance to the implementation of systems around a business process. When organizational forces of power and politics are strong, organizations are more likely to adopt systems that do not cross-organizational boundaries first. Taking the path of least organizational resistance means that organizations may ignore innovation dependencies and synergies in favor of ease of implementation and organizational costs. For example, a system that is completely contained within a department and is used only for internal processing is likely under full control of the department. This internal system will be much easier to implement than one that interfaces with people, systems, and processes outside of the department or organization. Therefore an organization or organizational unit will implement innovations in the internal stages of business processes first and then push them out. Hence, the organizational model suggests that patterns of adoption should be more rapid within internal departments than in systems where organizational boundaries are crossed.

\section{Proposition 1.}

Organizations are more likely to adopt systems that do not cross organizational boundaries before adopting systems that cross organizational boundaries.

\subsection{Patterns of adoption from an operational perspective}

An operations view on adoption of IT innovations produces patterns that focus on improving efficiency and on reducing cost and variance. This perspective is most useful when systems are used to reduce variation, increase or improve output, or decrease costs. The theory of constraints presented by Goldratt and Cox [22] applies in this situation and has two implications. First, a firm will look to bottlenecks or points of excessive variation in the process to start automation. Second, more efficiency is gained by reducing variation at the beginning of the process than at the end of the process. It is useful to discuss bottlenecks and points of excessive variation only in the context of specific processes. This view is consistent with that of [24] where recognition of the end-to-end business process flow is considered as an essential first step before business process change and IT interventions are even considered.

Because variation is produced at each stage of the business process, sequential automation may be the most effective at reducing variation and lowering costs. Referring to the theory of constraints [22], out-of-bound variation at the early stages of a process will cause subsequent stages to wait for acceptable output. This process of waiting is multiplied as each station introduces variation. Variation in business processes also tends to have a bullwhip effect, especially when those processes include imperfect information and forecasting. Therefore, reducing variation in the beginning stages of a business process will have a 
greater effect in decreasing the overall variation of a business process than will decreasing variation in the final stages of a business process.

Because the operational view of IT adoption sees the innovations as dependent of one another, one more argument becomes relevant for sequential adoption of innovations. If the relevant systems integrate with each other, interfaces often need to be developed or set up between systems. These interfaces are expensive to create and test. If systems are implemented in order, the number of interfaces to create for each system is minimized. If systems are implemented out of order, interfaces will need to be created for each system's current environment (either human interfaces or ad-hoc temporary solutions). Later, when the whole process is automated those interfaces will have to be abandoned and new interfaces implemented for the new environment. This argument is based on the assumption that systems in the business process integrate with each other.

Considering the effects of variation, variation detection and interface implementation costs, we predict that automating a process sequentially from the beginning of the process to the end is effective at reducing costs, improving output and reaching business goals. This assertion is made with the recognition that in the presence of bottlenecks and points of unusual variation, order of adoption will vary. We label this the operations model.

\section{Proposition 2.}

Sequence of systems adoption is likely to reflect the temporal ordering of the business process steps.

\subsection{Potential impacts of operational and organizational models on financial outcomes}

Because the organizational model of adoption focuses on appeasing political actors and the operations model of adoption is focused on reducing variance and improving quality and efficiency in an end-to-end business process flow, performance implications manifest in both instances. Where political actors are powerful, an organization may find the savings of appeasing these actors greater than the benefits provided by following an operational model of adoption. When this is the case, we should expect to see more organizational adoption and lower costs associated with the organizational model of adoption than with the operational model of adoption. In other cases, political actors may not have the power to use financial resources or create inefficiencies to oppose system adoption. In these cases, savings accrued from following an operational model of adoption are likely to be greater than those generated by appeasing the political 
actors. In this case we should see lower costs associated with the operational model of adoption than with the organizational model of adoption (Fig. 1 and Fig. 2).

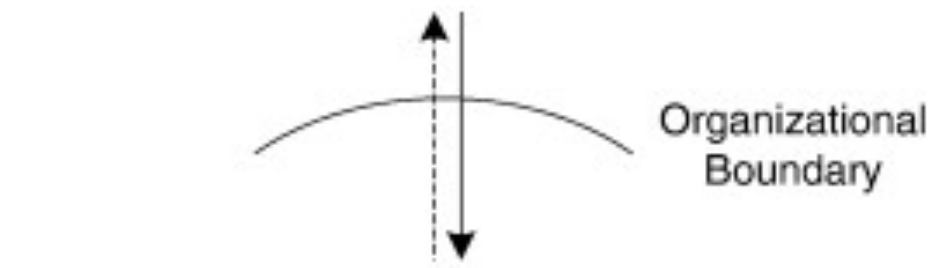

Start Here
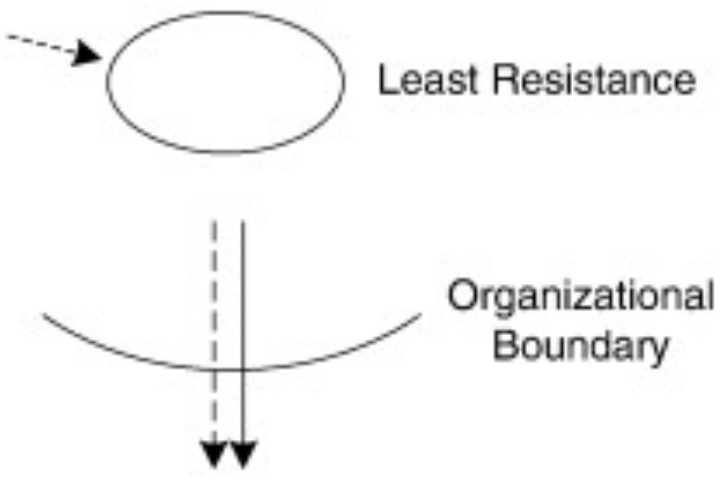

Business Process Flow

- - - Pattern of Adoption of Systems

Fig. 1.

Organizational model of adoption. 


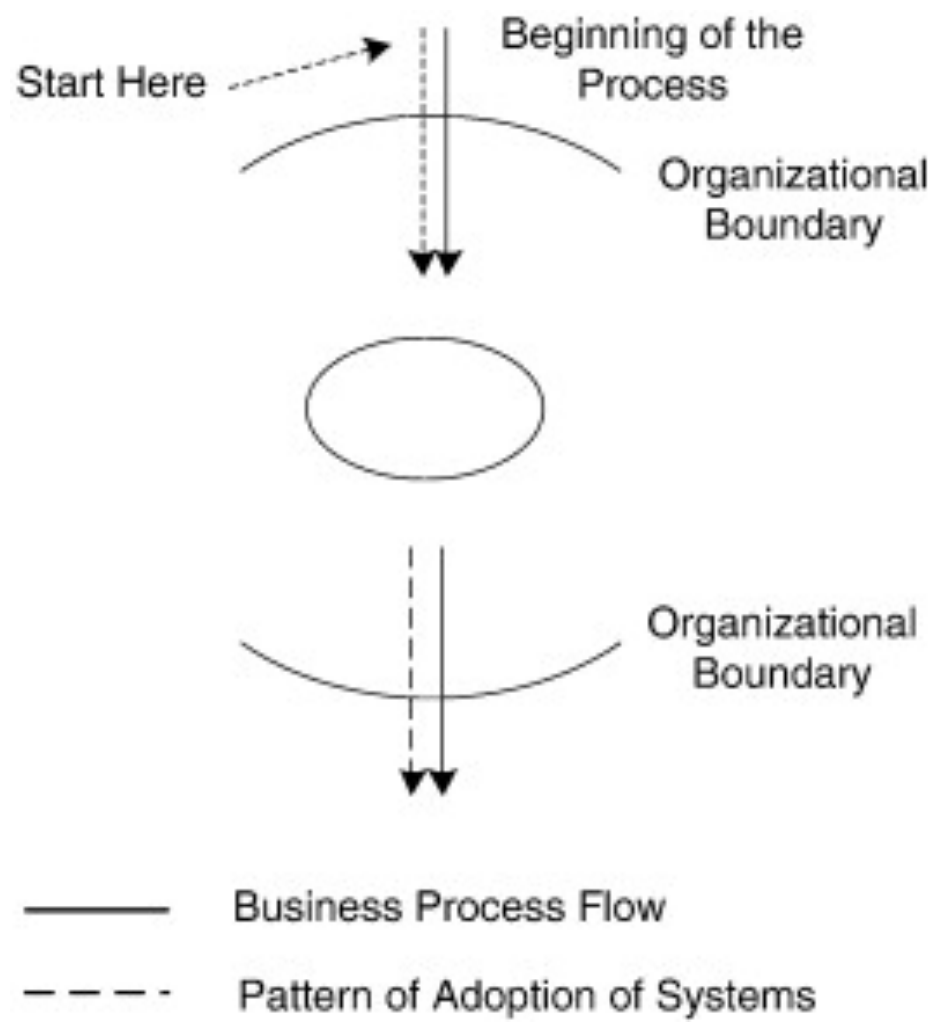

Fig. 2.

Operational model of adoption.

The conflict between operational and organizational models of adoption is highly relevant in the healthcare context. In healthcare organizations it is likely that stakeholders exert influence on how technologies are adopted. This is especially so in the case of physicians, who have considerable autonomy in their interactions with hospitals. In many cases, physicians are affiliated with hospitals and not directly employed. Nurses and pharmacists also exert considerable influence in adoption processes. It is well documented that nurses can create workarounds in clinical processes to adapt to the introduction of technology in their processes [39]. Workarounds can create inefficiencies in the care process that would ultimately impact organizational performance. 
Based on issues detailed for the organizational and operational processes, we conjecture that there are organizational performance implications from IT adoption in business processes and propose:

\section{Proposition 3.}

Different models of technology adoption are associated with dissimilar financial outcomes.

We examine data relating to the medication management process to look for evidence in support of these hypotheses. The next section describes the context and examination of the operational and organizational models of adoption.

\section{THE MEDICATION MANAGEMENT CONTEXT}

This study focuses on the process of medication prescribing and dispensing within a hospital. The medication process within hospitals is an issue of significant interest to policymakers and managers. Strong evidence has documented high rates of medication errors resulting from wrong drug or dosage administered to patients [11]. Information technology is widely viewed as an essential tool to improve medication safety by automating these business processes [7]. This process is also described in Bates [6] and focuses on the core of the business process and on the systems connected to administering medications and monitoring patients. We focus on the core process of creating the order to producing and delivering the packaged prescription to the clinician.

The process is initiated when a physician places an order for medication. The nurses on the floor attend to patient's needs and implement the doctor's orders. Nurses add notes to the medical chart describing assessments, interventions (including medications), and the response of the patient. When pharmacies receive physicians' orders they check the orders against the patient's charts and records to avoid any adverse effects such as drug interactions or allergic reactions. If the prescription meets the pharmacist's standards, the order is then processed. The medication is measured and mixed or counted in the pharmacy. It is then packaged and sent to the floor nurses for administration.

Variations in this process can occur for several reasons. An example deviation is in the case of controlled substances. Often the pharmacy will keep an automated dispensing machine on the unit floor. Nurses have access to this unit as granted by the pharmacy. The unit records who opened the unit, how long it was open and how much medication was taken. Other medications which are standing orders or common medications may be kept on the unit floor. 
From an organizational perspective this process involves three distinct groups with complementary tasks: physicians prescribe, pharmacists dispense, and nurses administer medications and record effects. Information systems supporting these groups are typically defined within defined functional boundaries. Additional systems are needed to span organizational boundaries and connect the business processes represented by these groups in an end-toend fashion. From an operational perspective the medication process can be viewed as a series of tasks initiated by the physicians' inputs (the prescriptions). The process continues with verification and dispensing by the pharmacist. The process ends with administration and recording of the event by the nurse.

Six systems that support the three-stage process of prescribing, dispensing, and administration (see Fig. 3) are included in this study. A physician's order can be generated either by nurses entering it into the order communication and results (OCR) system or directly when physicians use a computerized physician order entry (CPOE) system. The CPOE system requires greater involvement of physicians in the process since it requires direct inputs on a computer or handheld device. This is important to note because physicians are not typically hospital employees and usually work outside of the organizational boundaries of the hospital. Clinical documentation (CD) system provides an electronic version of patient's care plan including their medication schedule. Thus, CD helps to facilitate the ordering of medications. The pharmacy processes the order using the pharmacy information system (PIS). PIS is used within the pharmacy department and does not cross organizational boundaries. Medications are then sent to the nurses or released using the automated dispensing machines (ADM). Finally, the administration of the medication is recorded in the electronic medication administration record (EMAR). For this study, we focus on the core operations of the medication management process and have excluded several supplementary systems related to this context - such as computerized patient records, clinical decision support, robot technology for dispensing, and bar coding. 


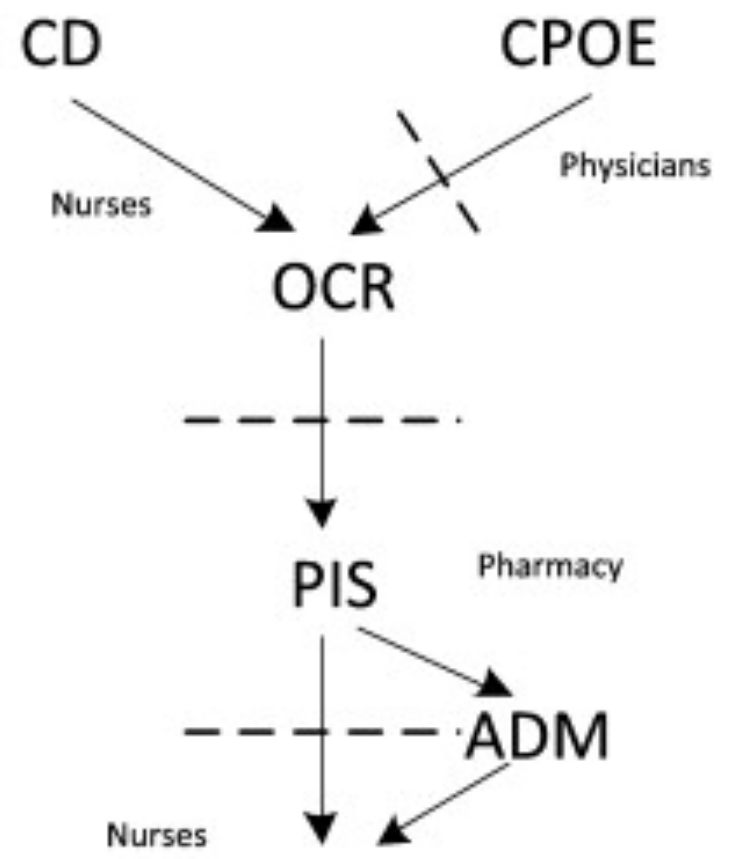

EMAR

\section{Business Process Flow \\ $--\cdots \cdot$ Organizational Boundaries}

Fig. 3.

The medication management process and systems.

To apply the operational and organizational models to the medication management process, we must discuss the characteristics of the process and the organizational environment around the process. The operational model of adoption as defined earlier is based on the process sequence. As indicated in Fig. 3, there are potentially two starting points for this sequence represented by CD or CPOE. In the case of organizational model of adoption, the process boundaries define the theorized sequences. The three stakeholder groups nurses, physicians and pharmacists define the organizational boundaries. While adoption can be initiated by any of the stakeholders, organizational realities [29] would suggest that physicians will be the most resistant to IT adoption. Though CPOE creates efficiencies for the nurses and hospital staff, physicians often find typing orders into the computer more cumbersome than writing a few lines of orders into the chart. In addition, physicians find CPOE inconvenient because the computer interface is rarely in your pocket or at your fingertips while you talk with the patient. CPOE also requires the doctor to log into the system and remember 
yet another password. The effects of these issues are increased by the fact that most doctors are not directly employed by the hospital. For this reason we propose that the CPOE will consistently appear last in the adoption sequence. Though nurses input information into EMAR, physicians are required to interact with the system. Therefore we predict that CPOE and the EMAR will be the most difficult systems to adopt. Given that nurses and pharmacists are employed by the hospitals, it is less likely that they will be able to resist process changes, even when the systems could increase the complexity of their work. However, if crossing organizational boundaries is more difficult than working within a single organization, OCR and ADM would be more difficult to adopt than would PIS and $\mathrm{CD}$. We therefore define four distinct sequences for the operations model of adoption and four distinct sequences for the organizational model of adoption (see Table 1).

Table 1

Operational and organizational reference sequences.

\begin{tabular}{lllllll}
\hline Model & \multicolumn{9}{l}{ Order of adoption } \\
\cline { 2 - 7 } & 1 & 2 & 3 & 4 & 5 & 6 \\
\hline Operational patterns & CPOE & CD & OCR & PIS & ADM & EMAR \\
& CD & CPOE & OCR & PIS & ADM & EMAR \\
& CPOE & CD & OCR & PIS & EMAR & \\
Organizational patterns & CD & CPOE & OCR & PIS & EMAR & \\
& CD & PIS & OCR & ADM & EMAR & CPOE \\
& PIS & CD & OCR & ADM & EMAR & CPOE \\
& CD & PIS & ADM & OCR & EMAR & CPOE \\
& PIS & CD & ADM & OCR & EMAR & CPOE \\
\hline
\end{tabular}

To look for support for the financial proposition, we use operational costs, operational revenue, and net income per patient day. Dividing net income and operational costs by patient day standardizes the measure across hospitals of varying sizes. The medication management process spans multiple organizational units and political actors. This fact makes the use of individual cost estimates such as pharmacy salaries or nursing salaries difficult to use. Many doctors are not paid by the hospital. Many costs of organizational adaptation will occur in unpredictable places such as concessions offered to one party to adopt the system, which may be unrelated to the system itself. Therefore hospital operational costs are the closest financial predictor to the costs associated with the medication management process. However, operational costs only describe part of the picture. Increased costs may be justified if increased revenues are larger. Therefore we have also included operating revenue and net income. 


\section{DATA AND RESEARCH METHOD}

Our dataset provides a multi-year view to the industry. Additionally, given the business process context of this study, regulation of medication prescription and administration allows for a common understanding of the business processes involved. To evaluate the organizational and operational influences on the sequence of IT adoption, we evaluate our theorized sequences for the two orientations using a sequence analysis method developed in the sociology and genetics contexts [2]. We then estimate the effect of each orientation on hospital financial outcomes.

\subsection{Data}

The adoption data is constructed from the 1998-2007 HIMSS Analytics database. The HIMSS database provides information on which systems were adopted by over 5000 hospitals. In many cases, there is also information on the year each system was adopted. When contract date and implementation information are not available in any database, it is often possible to determine in exactly what year the system was adopted by looking at the automation status provided by each database. When it is not possible to determine the exact year of adoption of each system, we dropped the hospital from our sample because this analysis depends on an accurate description of the order of adoption. Dropping hospitals for which exact order cannot be calculated leaves 2156 hospitals. The adoption data had to be further reduced by removing observations in which a hospital adopted more than one of the systems of interest in a single year. 1490 hospitals are removed which adopted more than one system in a year. To ensure that we could interpret the hospital's adoption pattern as a path, we only considered hospitals which had adopted three or more systems. Almost half of the remaining hospitals have not automated any of the systems of interest. Another 124 hospitals had adopted only one or two systems. Of the remaining 188 hospitals, 140 match to the dependent variables which are taken from other sources.

Hospital characteristics and financial data on net income, operating revenues, and operating expenses were collected from 2007 databases from the American Hospital Association and the Center for Medicare and Medicaid Services. Bias in hospital demographics is due to the fact that only hospitals with sequential adoption of medication management systems may be included. The sample represents hospitals which are larger than the national average. Hospitals in the sample provide more services (represented by the technical index), are more often not-for-profit, and are more likely to be a member of a multiple hospital system (see Table 2). 
Table 2

Descriptive statistics and population-sample comparison.

\begin{tabular}{lll}
\hline & AHA population & Sample \\
\hline $\mathrm{N}$ & 6312 & 140 \\
Beds & $156(181)$ & $187(161)$ \\
Medicare payer mix & $44.18(20.52)$ & $45.85(13.46)$ \\
Medicaid payer mix & $15.16(11.94)$ & $17.38(10.73)$ \\
Technical index & $2.56(2.37)$ & $3.42(2.35)$ \\
JCAHO & $71.4 \%$ & $77.9 \%$ \\
Not-for-profit & $50.1 \%$ & $74.3 \%$ \\
For-profit & $23.8 \%$ & $6.4 \%$ \\
Government & $26.2 \%$ & $19.3 \%$ \\
System member & $55.1 \%$ & $68.6 \%$ \\
COTH membership & $5.8 \%$ & $8.6 \%$ \\
Assoc. w/med school & $23.2 \%$ & $30.0 \%$ \\
Metro area & $64.6 \%$ & $64.3 \%$ \\
Micro area & $15.5 \%$ & $21.2 \%$ \\
Rural area & $19.8 \%$ & $14.3 \%$ \\
System count ${ }^{2}$ & - & $4.24(1.04)$ \\
Operational distance & - & $0.748(0.149)$ \\
Organizational distance & - & $0.381(0.206)$ \\
\hline
\end{tabular}

${ }^{2}$ Only includes a count of the six systems of interest.

\subsection{Event sequence analysis}

The need for event sequence analysis arises in many social and scientific studies [10]. Sequence ordering allows one to investigate the influence of variables in the sequences and if/how a specific pattern of events represents the context and the process [2]. This technique has been used in natural and social sciences including analysis of DNA sequences [34], study of ritual dances [1] and the study of careers of 18th century musicians [2]. In our research context, sequence ordering of IT adoption is temporal and discrete. Each event in the sequence signifies the adoption of an IT system in the process.

Sequence construction and comparison require the use of dynamic programming methods to calculate the distances between sequences. In our study, we used the $S Q$ package in Stata to calculate distances between patterns. The $S Q$ package is described in Brzinsky-Fay et al. [10]. This package calculates the Levenshtein distance between observed and reference sequences. The Levenshtein distance was first developed to calculate the distance between two strings of characters [28]. 
The Levenshtein distance calculates the number of operations (insertions and deletions) to transform one sequence into another. As applied in this context, the maximum number of operations to transform any sequence is six. The count of operations for each hospital is then standardized to a scale of 0 to 1 . This means that each operation increases the distance by 0.1667 . The context of adoption of multiple systems requires that we also account for different lengths of the sequence. Therefore we calculated the difference between the first four systems of a given adoption model and the hospital adoption path if the hospital had only adopted four systems. The implication of this is that if the hospital has followed the adoption model perfectly to this point, their score would be zero. It is therefore important to include the system count and an interaction term between system count and the adoption model distance in the regression.

To calculate an example distance requires a reference pattern (from the operational model) and a pattern followed by several hospitals in our sample:

$$
\begin{aligned}
& \text { Sample pattern: } \mathrm{PIS} \rightarrow \mathrm{CD} \rightarrow \mathrm{OCR} \rightarrow \mathrm{ADM} \rightarrow \mathrm{EMAR} \rightarrow \mathrm{CPOE} \\
& \text { Reference pattern: } \mathrm{CD} \rightarrow \mathrm{CPOE} \rightarrow \mathrm{OCR} \rightarrow \mathrm{PIS} \rightarrow \mathrm{ADM} \rightarrow \mathrm{EMAR} .
\end{aligned}
$$

In the reference pattern CD should occur first and PIS should occur after CD and OCR. This can be done with one deletion and one insertion.

$$
\text { Transformation after step 1: CD } \rightarrow \text { OCR } \rightarrow \text { PIS } \rightarrow \text { ADM } \rightarrow \text { EMAR } \rightarrow \text { CPOE. }
$$

Further, CPOE should occur before PIS and not at the end of the sequence. This can again be corrected with one deletion and one insertion.

Transformation after step $2: \mathrm{CD} \rightarrow \mathrm{CPOE} \rightarrow \mathrm{OCR} \rightarrow \mathrm{PIS} \rightarrow \mathrm{ADM} \rightarrow$ EMAR.

After two insertions and two deletions, the sample pattern now matches the reference pattern for a total of four operations. Given that the greatest distance between any hospital's pattern of adoption and the reference patterns was six, the hospital would receive a distance to this reference of $4 / 6=0.667$.

To complete the analysis, the data must be shaped into long form with each record containing the hospital identifier, the name of the system which was adopted and the order in which that system falls. After processing the data through the SQ package, it can then be transformed back to short form and analyzed with traditional statistical tools. Because of the existence of multiple organizational units and branches in the process, both models of adoption are associated with multiple reference patterns. To correct for this, we calculated the Levenshtein distance for each hospital against all reference patterns. The smallest measurement obtained from comparisons to the reference patterns associated with the operational model is used as the operational distance. The same was done for the organizational distance. Using multiple reference patterns 
makes it possible to examine a more complex business process. Nevertheless, it will bias the analysis against finding significant results because using the lowest measure substantially reduces variance.

Further, we note that if a hospital was following the operation or organizational model, but had not completed the sequence, it receives a perfect score. The subsequent regression should therefore account for the number of systems adopted and for a potential interaction between the distance measure and the number of systems the hospital had adopted. Our data contain 56 different adoption patterns. The most common pattern of adoption is OCR, PIS, then ADM. (see Table 3, Fig. 4 and Fig. 5).

\section{Table 3}

Five most common patterns of adoption.

\begin{tabular}{lc}
\hline Sequence-pattern & Freq. \\
\hline $\mathrm{OCR} \rightarrow \mathrm{PIS} \rightarrow \mathrm{ADM}$ & 18 \\
$\mathrm{PIS} \rightarrow \mathrm{OCR} \rightarrow \mathrm{CD} \rightarrow \mathrm{ADM}$ & 12 \\
$\mathrm{OCR} \rightarrow \mathrm{PIS} \rightarrow \mathrm{ADM} \rightarrow \mathrm{CD}$ & 8 \\
$\mathrm{PIS} \rightarrow \mathrm{OCR} \rightarrow \mathrm{ADM}$ & 7 \\
$\mathrm{OCR} \rightarrow \mathrm{CD} \rightarrow \mathrm{PIS} \rightarrow \mathrm{ADM} \rightarrow$ EMAR & 6 \\
\hline
\end{tabular}




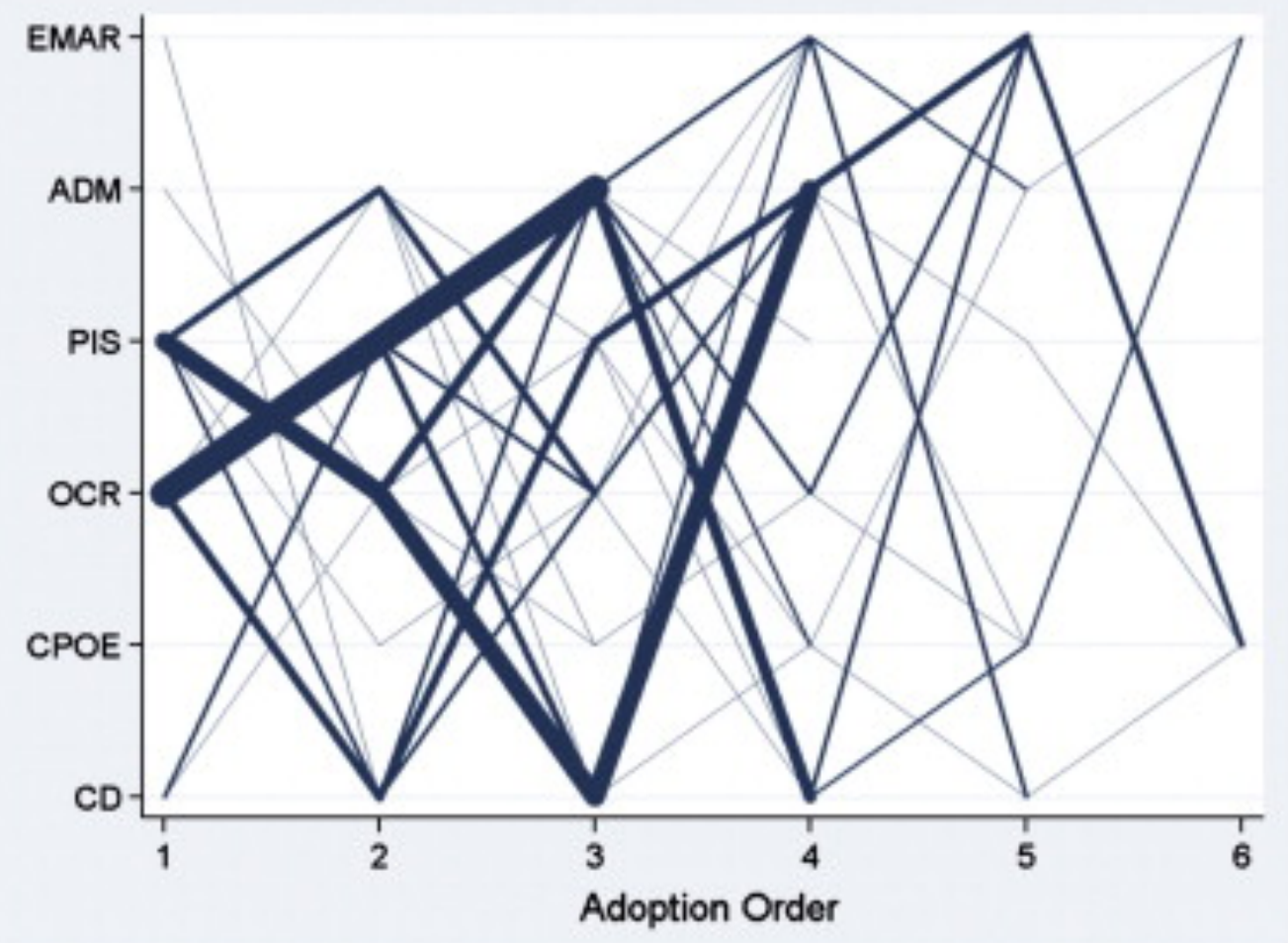

Fig. 4.

Parallel-coordinates plot. 


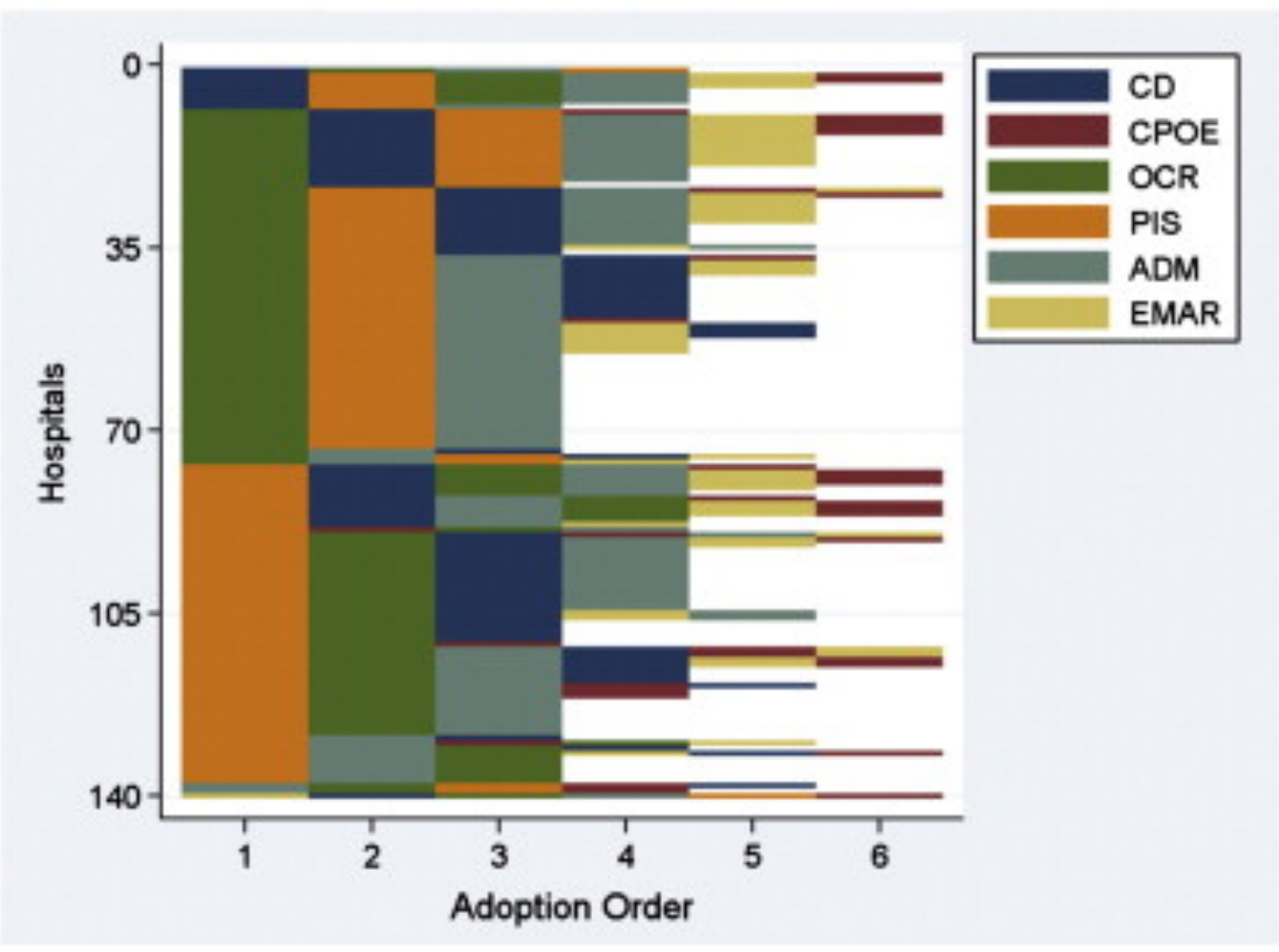

Fig. 5.

Sequence index plot.

The number of applications of interest adopted by the hospital has a normal distribution. This suggests that we are seeing hospitals at a variety of different stages in the adoption process. The six systems in this study provide a range of characteristics. The most commonly adopted system is PIS followed by OCR. The relative diffusion of these applications may have to do with the age of the innovation. The earliest adoption of CPOE is nine years after the earliest adoption of the next newest technology. The earliest contracts for all of the other technologies are in the 1970s.

In this context, sequence analysis provides a standard measure for how close each hospital's adoption path is to the operational and organizational models of adoption. This method provides a novel and valuable perspective through which we can view the adoption of a set of systems. Using traditional methods, we cannot differentiate between two hospitals which currently may have the same application set, but had come to this point in different paths. Sequence analysis allows us to look at the effects of different paths of adoption. 


\section{RESULTS}

To illustrate and evaluate the viability of our propositions it is important that we have a common understanding of the measurement for organizational and operational distances as well as regression of these distances on the outcome variables (presented in Table 4).

\section{Table 4}

Robust regressions on net income, operating cost, and operating revenue.

\begin{tabular}{|c|c|c|c|}
\hline DV & $\begin{array}{l}\text { Adjusted net } \\
\text { income }\end{array}$ & $\begin{array}{l}\text { Adjusted operating } \\
\text { expense }\end{array}$ & $\begin{array}{l}\text { Adjusted operating } \\
\text { revenue }\end{array}$ \\
\hline $\begin{array}{c}\text { Operational } \\
\text { measure }\end{array}$ & $-1347.36^{* * * *}$ & -2361.44 & $-3585.03^{* * *}$ \\
\hline $\begin{array}{l}\text { Operational*sys } \\
\text { count }\end{array}$ & $295,05^{* k * *}$ & 404.15 & $681.74^{*}$ \\
\hline $\begin{array}{l}\text { Organizational } \\
\text { measure }\end{array}$ & $932.20^{\text {*kk* }}$ & $2864.06^{*}$ & 2424.93 \\
\hline $\begin{array}{l}\text { Organizational*sys } \\
\text { count }\end{array}$ & $-196.99^{k-k \neq k}$ & $-588.00^{*}$ & -492.62 \\
\hline System count & $91.169^{*}$ & 300.86 & 154.19 \\
\hline Ln(Beds) & 6.35 & -117.04 & -71.97 \\
\hline $\begin{array}{l}\text { Medicare payer } \\
\text { mix }\end{array}$ & $-5.32^{\text {*k* }}$ & $-17.78^{\text {*k** }}$ & $-19.67^{* * * *}$ \\
\hline $\begin{array}{l}\text { Medicaid payer } \\
\text { mix }\end{array}$ & $-3.18^{* * *}$ & -7.22 & $-11.16^{*}$ \\
\hline Technical index & 0.05 & 36.61 & 40.13 \\
\hline $\begin{array}{l}\text { JCAHO } \\
\text { accreditation }\end{array}$ & -31.38 & $288.85^{* * *}$ & $395.59^{* k * *}$ \\
\hline Government & 9.05 & -81.66 & -210.85 \\
\hline For-profit & -23.81 & -316.77 & -242.00 \\
\hline System member & $57.59^{* * *}$ & $258.51^{* *}$ & $361.21^{\text {****** }}$ \\
\hline COTH member & 61.97 & $716.28^{\text {*ak* }}$ & $796.41^{\text {k*k* }}$ \\
\hline $\begin{array}{l}\text { Assoc. w/med } \\
\text { school }\end{array}$ & 24.16 & 168.45 & 125.40 \\
\hline Rural location & 42,00 & $-319.77^{*}$ & -84.89 \\
\hline Constant & -64.31 & 1226.34 & 1654.91 \\
\hline $\mathrm{N}$ & 140 & 140 & 140 \\
\hline $\mathrm{F}(16,123)$ & 4,39 & 6.48 & 7.88 \\
\hline Prob $>F$ & $<0,001$ & $<0.001$ & $<0.001$ \\
\hline R-square & 0.2368 & 0.3544 & 0.4035 \\
\hline
\end{tabular}


On average, the Levenshtein distance from the organizational model is only 0.381 as compared to the 0.748 distance from the operational model. This is a statistically significant difference with a p-value of less than 0.001 . The first two propositions suggest that organizational and operational models of adoption exist. This difference shows that hospitals are more likely to follow the organizational model of adoption. Therefore, there is more evidence to suggest that the organizational model of adoption is followed by hospitals than the operational model of adoption.

The operational and organizational distances provide two other helpful insights. First, operational and organizational distances showed no evidence of correlation (Pearson coefficient < 0.01). This suggests that the two patterns (or sets of patterns) are distinct. A low correlation coefficient also suggests that we may include both in the same model. Second, the underlying theory suggests that these measures might be correlated with organizational size. Simple correlations do not support this conclusion. P-values of Pearson coefficients of the relationship between beds and the distance measures are insignificant. This finding is further confirmed by the regressions. From this point forward we have reversed the distance measures for ease of interpretation (1-operational distance = operational measure).

A more rigorous look at the data related to the three propositions and distances to the reference patterns can be conducted using regression. Because of the presence of outliers we elected to use a weighted least squares regression (see Table 4). This method is an iterative process which puts less weight on observations which dramatically change the coefficients. Both net income and operating costs were divided by the number of patient days for each hospital. This gives an estimate of hospital net income, operating costs, and operating revenue per day each patient is in the hospital. Because operating costs are theoretically closer to the expected outcomes of automating the medication management process, it was expected that the R-squared would be higher than for net income or operating revenue.

R-square values calculated for the WLS regressions are reasonably high $(0.24$, 0.35 , and 0.40 ). Overall $p$-values show that all three regressions are statistically significant. The standard control variables used in relation to hospital financial outcomes show some significance. Higher Medicare payer mix is associated with lower net income, operating expense and operating revenue per patient day. JCAHO accredited hospitals incur more costs, but have higher compensating revenues. Being a member of system of hospitals or a COTH member has a similar effect. The regression models appear to fit the data well.

The coefficient of the organizational measure regressed on net income is significant with a p-value of less than 0.01 . Interpreting the coefficients related to the model measures requires dividing the measures by six. In this case, the largest number of changes to bring the model into compliance with the reference 
pattern is six. Therefore, each step following an organizational model of adoption is associated with approximately $\$ 155$ increase in net income on average per patient day. For large hospitals which serve many patients, this outcome is substantial. As a hospital progresses along a model, the effect is reduced by about $\$ 33$ dollars with each additional system. These diminishing returns suggest that the effect of following the operational model disappears when the process is fully automated.

The coefficient of the operational measure regressed on net income is also significant. Each step following an operational model is associated with about $\$ 225$ decrease to net income per patient day. As with the organizational model, this is diminished as a hospital progresses along the pattern of adoption. Each additional system is associated with approximately $\$ 50$ reduction per patient day. Therefore the effects on net income seem to disappear as the hospital progresses toward full automation. A reasonable interpretation of the organizational and operational effects is that if two similar hospitals follow different patterns, the effect on net income may be the same after full automation. Nevertheless, a hospital which follows an organizational pattern of adoption will see more financial benefits during the process of automation than a hospital which followed the operational model.

The regressions support Proposition 3 and lend support to the assertion that different financial outcomes are likely to be associated with different adoption patterns. In order to examine this proposition, linear combinations of the coefficients related to the organizational measure and the operational measure were conducted. The $p$-value for the difference between organizational and operational measures in the net income regression was $<0.01$. In the operating expense regression the $p$-value was 0.03 . In the operating revenue regression the $p$-value was 0.01 . At $p<0.05$, all three tests show that the differences in the coefficients of operation and organizational measures are statistically significant. The organizational model is positively correlated with net income and operating revenue. The operational model is negatively associated with operating expense. If net income is considered inclusive of operating revenue and operating expense, the organizational model is related to a more desirable financial outcome in this context.

Finally, we evaluated the Levenshtein distance in regards to discriminant validity in earlier versions of the analysis. To do this, the reference sequences were redesigned and examined in two ways. The first was to randomly order the systems in the sequence. The second was to deliberately change organizational boundary-spanning systems with internal systems and place the systems in an order which removed almost all of the relationships defined by the process as developed in Section 4. The effects of the measures of distance on the dependent variables disappeared. 


\section{DISCUSSION}

This section first discusses the support for the three propositions. Findings related to different financial outcomes are interpreted. Then managerial implications are discussed. Finally, implications and future research directions are laid out.

The analysis provides support for all three propositions. The first two propositions are supported in the evaluation of the organizational and operational measures as well as by the evaluation of discriminant validity. The first two propositions posit that organizational and operational patterns do exist in the data. Examples of hospitals which followed one pattern or the other very closely are observable in the data. Comparison of the two measures suggests that the organizational model of adoption is observed more often in the data. The tests of discriminant validity supported the theory behind the existence of the organizational and operational patterns. The tests showed no results when randomizing the order of the reference sequence and when deliberately organizing the reference sequence to remove proposed relationships. The prevalence of the organizational pattern makes sense. According to Casalino et al. [13], physicians often do not want to align with the needs of hospitals. Many doctors are refusing to perform voluntary hospital duties. They show that there is a group of physicians that align with the hospital given pressures of rent and malpractice insurance. Nevertheless, a large number of physicians spend great energy not to align with hospitals and hospitalists. Organizational issues are strong in daily operations of the hospital.

The final proposition was supported using linear combinations of the effects related to organizational and operational measures of adoption. The third proposition posits that the two models of adoption will be associated with different financial outcomes. For all three cases (adjusted net income, adjusted operating expenses, and adjusted operating revenue), the operational measure was significantly different than the organizational measure at $p=0.05$. Two of the measures are statistically significant at alpha $=0.01$.

The analysis showed different financial outcomes associated with the organizational and operational models of adoption. Notably, the different outcomes associated with these two models diminish and possibly disappear once the whole process has been automated. These diminishing returns for adoption of each incremental system along the pattern suggest that there may not be long term financial differences among hospitals which follow different patterns of adoption. Nevertheless, significant savings can accrue over the time period when automation progresses through the different parts of the process. Following the organizational model of adoption is associated with approximately $\$ 155$ dollar increase in net income per patient day. In the study sample, mean for patient-days in a hospital is over 69,000 . Thus, increase in net income per patient day could translate to millions of dollars for the average hospital in a single year. 
Following the operational model of adoption is associated with approximately $\$ 225$ dollars decrease in net income per patient day. Again, recognition that this effect diminishes with the adoption of each additional system is important in order not to overstate the effects. Even with that recognition, these are substantial financial effects for hospitals going through the process of automating their medication management systems.

While our analysis does not suggest that there is a causal relationship between the adoption patterns and financial outcomes, it is likely that other factors such as management's willingness to keep employees satisfied are related to the organizational measure of adoption. Any discussion of possible relationships can be complicated and nuanced. It might be that adoption according to an operational pattern helps to reduce operating costs. Nevertheless, in an environment such as the hospital, keeping the employees satisfied has a significant impact on demand for services at a given hospital. Such ideas must be tested further by studies designed to test causal relationships.

We also find that the adoption patterns of hospitals in our sample are closer to the organizational model of adoption than to the operational model of adoption. Several actors in the medication management process have significant political power. Physicians are not often employed by the hospital. Hospitals must comply with the manner in which physicians wish to work (to some extent) or the physicians can take their patients to another facility. Further research on the effect of adoption patterns should study a business process in which politics and power are not as much a part of the decision process.

Implications of this study for decision makers and managers are three-fold. First, there are unforeseen financial effects of cost cutting in this context, particularly if cost cutting is implemented through a variance control approach. Second, both operational and organizational models suggest strategies for long-term decision making. Finally, organizations that find themselves on the wrong path are not at a permanent disadvantage.

One of the surprising results of the analysis is that actions related to cost cutting may lead to negative impacts on the bottom line. An operational approach to adoption means that decision makers are focused directly on quality control and reducing variance. The effectiveness of the operational approach is visible when comparing costs related to the two patterns of adoption. Operating expenses are significantly higher in relation to organizational patterns of adoption than in relation to operational patterns of adoption. This is predicted by the theory. The surprise comes when comparing net income. Following an organizational pattern of adoption is associated with higher revenues and higher net income. These findings support the idea that greater financial viability is associated with the organizational pattern of adoption. 
The second set of implications for decision makers relates to strategies for adoption of hospital IT. Long-term planning involves deciding which systems will be implemented first. The importance of the organizational pattern of adoption within the hospital context suggests that implementation should first be completed where the hospital has most control. In the context of this study, the pharmacy systems should be implemented first, and then systems related to nursing. Systems that require physician training and interaction should be implemented last. This is particularly true in the traditional model of the hospitalphysician relationship. Although the effect would not be as strong in the context of hospitalists, it is our opinion that order of implementation would still hold because of the power of hospitalists within the hospital. This idea deservers further investigation in future research.

Nevertheless, decision makers should not ignore the operational model in longterm planning. The analysis shows that the organizational model and operational model are not mutually exclusive. Although the organizational model is associated with more favorable financial outcomes, certain contexts may dictate the use of an operational approach to systems implementation. The operational model of implementation was associated with less operational expenses than was the organizational model. When several process-related systems exist within one department, such as the hospital pharmacy, systems should be implemented in the order of the steps of the business process. This will control variance and improve quality at the earliest possible points in the process and thus reduce operating expenses. Decision makers must note that although these ideas are supported by the analysis, urther testing in other contexts is necessary to provide conclusive evidence.

Finally, the analysis provides an optimistic perspective for decision makers who may be experiencing unexpected negative financial outcomes from following an operational approach to implementation of hospital systems. The negative financial impacts of following a pure operational model diminish as the organization implements more of the process. The positive financial impacts related to the organizational pattern of adoption also diminish as the process becomes completely automated. If this relationship holds up to further testing, it would mean that these financial outcomes are temporary. While substantially better financial outcomes are associated with the organizational model, following one path or the other is not associated with a significant long-term financial advantage.

This whole-process perspective has implications for research in regards to the value of IT. The findings suggest that the relationships among the systems are an important consideration in regards to IT value. Most research regarding the value of IT observes a single system (e.g. [16]), calculates the individual effects or linear effects of the count of systems (e.g. [14]), or uses aggregated measures of IT (e.g. [33]). Although research IT value studies have increased our knowledge of how IT creates value, a clearer picture of how IT creates value can 
be found by applying the whole-process perspective discussed in this paper. Not accounting for these effects could lead to biased results.

The Levenshtein distance made this study possible in two ways. The first is a calculation of distance between hypothesized patterns and observed patterns. The Levenshtein distance has been used and validated in multiple disciplines [e.g. [1], [2] and [34]]. Few other validated measures exist. Network analysis, as implemented in [26], was considered as one alternative. Treating systems as nodes and relationships as edges, these network tools would allow for multiple adoptions in a single year. However, network analysis becomes impractical in solving the second issue that is resolved by the Levenshtein distance.

Operationalizing the hypothesized models within the context of a complex process creates multiple instances of the perfect sequence (as seen in Table 1). When systems are implemented in the same period, the number of permutations becomes impractical. Further, as the number of permutations increases and the complexity of the network of systems increases, the threat to discriminant validity grows. It becomes more difficult to say that the operationalized patterns belong to only one theoretical model. Although the application of the Levenshtein distance has limitations, it makes this novel approach possible.

Finally, several other adoption phenomena are not accounted for in this work. The sample and analysis are limited to hospitals which had pure sequential adoption of the systems of interest. The theory has implications for co-adoption and big-bang techniques. Co-adoption may also be further related to financial outcomes because of the necessity to coordinate all actors [31], mass customization of vendor software [9], and/or the adaptation of large groups of processes to work with the system [5].

\section{CONCLUSION}

This study makes four main contributions. The business process is applied in a novel and unique manner to dictate how systems should integrate and be related. This application provides a new perspective and foundation for the development of heuristics for decision makers and for development of future research on integrated and related systems. The second contribution is the development of organizational and operational models of process automation. These models of adoption explain why different patterns of adoption may occur among similar entities. In this research we posit different financial outcomes in regard to different patterns of adoption. The third contribution is the application of sequence analysis and the Levenshtein distance to information systems research and the order of adoption of IS. Fourth, implications of this research impact IT planning within hospitals as well as IT strategy more generally. In relation to IT strategy, this research suggests the order in which a set of systems should be implemented. Within the hospital context, organizational patterns of adoption are related to better financial outcomes. This research suggests that hospitals which 
effectively reduce costs using an operational pattern of adoption may experience other negative financial impacts which outweigh any reduction of costs. Finally, the research suggests that hospitals which follow a path to automation associated with worse financial outcomes may not be at a permanent financial disadvantage because the negative effects are diminished as processes are completely automated.

Using business process, operations management, and organizational theory we have proposed an operational model of adoption which prioritizes system adoption based on business process requirements. Next, with organizational theory and information systems literature as a basis we developed an organizational model of adoption that prioritizes system adoption to fit organizational structure and politics. These competing models provide two perspectives often considered when explaining IT adoption in organizations. Our analysis suggests that in hospitals the organizational model tends to be the more significant explanation for complementary IT systems adoption.

This study leverages event sequence analysis to study the path of adoption of a set of systems. We use sequence analysis to calculate distances between actual adoption patterns and the theorized operational and organizational adoption patterns. We find evidence that different paths of adoption are related to different financial outcomes. In the hospital environment we find that following the operational model of adoption is associated with lower operating expenses. However, following the organizational model of adoption is associated with higher operating revenue and higher net income.

This study begins to address questions regarding the nature of systems complementarities and the importance of the path of adoption. The operational model of adoption suggests that the main source of complementarities may be found in the passing of information and the integration of systems. The analysis notably shows that different financial performance is associated with different paths of adoption although the paths involve the same systems. Although the theory suggests that this may be due to organizational fit and attention to the process, the analysis cannot confirm this part of the theory. This paper lays the foundation for future work with the order of adoption and the study of why path matters. 


\section{REFERENCES}

[1] A. Abbott, J. Forrest, Optimal matching method for historical sequences, Journal of Interdisciplinary History 16 (3) (1986) 471-494.

[2] A. Abbott, A.Hrycak, Measuring resemblance in sequence data: an optimal matching analysis of musicians' careers, The American Journal of Sociology 96 (1) (1990) 144-185.

[3] A.E. Alter, Business alignment: the eternal priority, $\mathrm{ClO}$ Insight, March 22, 2007. Available at http://www.cioinsight.com/c/a/Past-News/Business-AlignmentThe-Eternal-Priority/. Last accessed on September 24, 2010.

[4] C.M. Angst, S. Devaraj, C.C. Queenan, Performance Effects Related to the Sequence of Integration of Healthcare Technologies, Production and Operations Management 20 (3) (2011) 319-333.

[5] A. Barua, C.H.S. Lee, A.B.Whinston, The calculus of reengineering, Information Systems Research 7 (4) (1996) 409-428.

[6] D.W. Bates, Using information technology to reduce rates of medication errors in hospitals, British Medical Journal (BMJ) 320 (2000) 788-791.

[7] D.W. Bates, A. Gawande, Patient safety: improving patient safety with information technology, New England Journal of Medicine 348 (25) (2003) 2.

[8] G. Battisti, M.G. Colombo, L. Rabbiosi, Path dependency and causality testing in the presence of complementarity effects: an application to the adoption sequence of an it related innovation and new work practice, in: 15th (EC)squared Conference, 2004, Marseille, France.

[9] P. Bingi, M.K. Sharma, J.K. Godla, Critical issues affecting an ERP implementation, Information Systems Management 16 (3) (1999) 7-14.

[10] C. Brzinsky-Fay, U. Kohler, M. Luniak, Sequence analysis with Stata, The Stata Journal 6 (4) (2005) 435-460.

[11] R.T. Burlton, Business Process Management: Profiting from Process, Sams, Indianapolis, IN, 2001.

[12] J.A. Buzacott, Commonalities in reengineered business processes:models and issues, Management Science 42 (5) (1996) 768-782. 
[13] L.P. Casalino, E.A. November, R.A. Berenson, H.H. Pham, Hospitalphysician relations: two tracks and the decline of the voluntary medical staff model, Health Affairs 27 (5) (2008) 1305-1314.

[14] S.D. Culler, J.N. Hawley, V. Naylor, K.J. Rask, Is the availability of hospital it applications associated with a hospital's risk adjusted incidence rate for patient safety indicators: results from 66 Georgia hospitals, Journal of Medical Systems 31 (5) (2007) 319-327.

[15] E. Davidson, D. Heslinga, Bridging the it adoption gap for small physician practices: an action research study on electronic health records, Information Systems Management 24 (1) (2007) 15-28.

[16] S. Devaraj, R. Kohli, Performance impacts of information technology: is actual usage the missing link? Management Science 49 (3) (2003) 273-289.

[17] M. Furukawa, T.S. Raghu, B.M. Shao, Electronic medical records and cost efficiency in hospital medical-surgical units, Inquiry 47 (2) (2010) 110-123.

[18] M. Furukawa, T.S. Raghu, B.M. Shao, Electronic Medical Records, Nurse Staffing and Nurse-Sensitive Patient Outcomes: Evidence from the National Database of Nursing Quality Indicators, Medical Care Research and Review 45 (4) (2010) 941-962.

[19] M. Furukawa, T.S. Raghu, B.M. Shao, Electronic medical records, nurse staffing, and nurse sensitive patient outcomes: evidence from California hospitals, Health Services Research 45 (4) (2010) 941-962.

[20] M. Furukawa, T.S. Raghu, T.J. Spaulding, A. Vinze, Adoption of health information technology for medication safety in U.S. hospitals, Health Affairs 27 (3) (2008) 865-875.

[21] D. Gans, J. Kralewski, T. Hammons, B. Dowd, Medical groups' adoption of electronic health records and information systems, Health Affairs 24 (5) (2005) 1323-1333.

[22] E.M. Goldratt, J. Cox, The Goal: A Process of Ongoing Improvement, North River Press, Great Barrington, MA, 1984.

[23] T.F. Golob, A.C. Regan, Trucking industry adoption of information technology: a multivariate discrete choice model, Transportation Research Part C 10 (3) (2002) 205-228.

[24] M. Hammer, J. Champy, Reengineering the Corporation: A Manifesto for Business Revolution, Harper Business Editions, New York, NY, 1993. 
[25] M. Klein, 10 principles of reengineering, Executive Excellence 12 (2) (1995) 20.

[26] G. Kossinets, D.J. Watts, Empirical analysis of an evolving social network, Science 311 (5757) (2006) 88-90.

[27] L. Lapointe, S. Rivard, A multilevel model of resistance to information technology implementation, Management Information Systems Quarterly 29 (3) (2005) 461-491.

[28] V. Levenshtein, Binary codes capable of correcting deletions, insertions and reversals, Soviet Physics Doklady 10 (8) (1966) 707-710.

[29] M.L. Markus, Power, politics, and MIS implementation, Communications of the ACM 26 (6) (1983) 430-444.

[30] M.L. Markus, D. Robey, Information technology and organizational change: causal structure in theory and research, Management Science 34 (5) (1988) 583-598.

[31] M.L. Markus, C. Tanis, P.C. Van Fenema, Multisite ERP implementations, Communications of the ACM 43 (4) (2000) 42-46.

[32] N. Menachemi, A. Chukmaitov, C. Saunders, R.G. Brooks, Hospital quality of care: does information technology matter? The relationship between information technology adoption and quality of care, Health Care Management Review 33 (1) (2008) 51-59.

[33] N. Menachemi, N. Hikmet, A. Bhattacherjee, R.G. Brooks, The effect of payer mix on the adoption of information technologies by hospitals, Health Care Management Review 32 (2) (2007) 102-110.

[34] S. Needleman, C. Wunsch, A General method applicable to the search for similarities in the amino acid sequence of two proteins, Journal of Molecular Biology 48 (3) (1970) 443-453.

[35] J. Peppard, P. Rowland, The Essence of Business Process Reengineering, Prentice-Hall, New York, NY, 1995.

[36] T.S. Raghu, A. Vinze, A Business Process Context for Knowledge Management, Decision Support Systems 43 (3) (2007) 1062-1079.

[37] R.O. Rupp, J.R. Russel, The golden rules of process redesign, Quality Progress 27 (12) (1994) 85-92. 
[38] M.H. Smith, D. Weil, Ratcheting up: linked technology adoption in supply chains, Industrial Relations 44 (3) (2005) 490-508.

[39] A.L. Tucker, S.J. Spear, Operational failures and interruptions in hospital nursing, Health Services Research 41 (3) (2006) 643-662.

[40] A. Vishwanth, S.D. Scamurra, Barriers to the adoption of electronic health records: using concept mapping to develop a comprehensive empirical model, Health Informatics Journal 13 (2) (2007) 119-134. 